\title{
Surgical strategies and outcomes of spinal ependymomas of different lengths: analysis of 210 patients
}

\author{
Clinical article
}

\author{
Teng-yu Li, M.D., ${ }^{1,8}$ Jun-sheng Chu, M.D., ${ }^{1}$ Yu-lun Xu, M.D., Рh.D., ${ }^{1}$ Jun Yang, M.D., ${ }^{1}$ \\ James Wang, M.D., ${ }^{2}$ Yu-Hua Huang, M.D., Ph.D., ${ }^{3}$ AiJ-Lie Kwan, M.D., Ph.D., ${ }^{4-6}$ \\ and Gui-Huai Wang, M.D., Ph.D., ${ }^{1,7}$ \\ ${ }^{1}$ Department of Neurosurgery, Beijing Tiantan Hospital, Capital Medical University, Beijing, China; \\ ${ }^{2}$ Department of Neurosurgery, Providence Hospital, Seattle, Washington; ${ }^{3}$ Department of Neurosurgery, \\ Kaohsiung Chang Gung Memorial Hospital and Chang Gung University College of Medicine, Kaohsiung, \\ Taiwan; ${ }^{4}$ Department of Surgery, College of Medicine, Kaohsiung Medical University, Kaohsiung, Taiwan; \\ ${ }^{5}$ Department of Neurosurgery, Kaohsiung Medical University Hospital, Kaohsiung, Taiwan; ${ }^{6}$ Department \\ of Neurosurgery, University of Virginia, Charlottesville, Virginia; ${ }^{7}$ Beijing Neurosurgical Institute, Capital \\ Medical University, Beijing, China; and ${ }^{8}$ Department of Neurosurgery, Beijing Dongzhimen Hospital \\ Eastern, Beijing University of Chinese Medicine, Beijing, China
}

Object. The aim of this study was to investigate the surgical strategies and outcomes for spinal ependymomas of different lengths.

Methods. The authors used data from 210 patients with spinal ependymomas (WHO Grades II and III) in this 10-year retrospective study (January 1999 to December 2008), dividing them into 3 different groups according to length (spinal ependymomas $<5 \mathrm{~cm}, 5-10 \mathrm{~cm}$, and $>10 \mathrm{~cm}$ ). All patients underwent tumor resection. The basic characteristics of the patients were reviewed and the functional status was assessed using the McCormick classification.

Results. There were 89,81 , and 40 patients, respectively, in the 3 groups $(<5 \mathrm{~cm}, 5-10 \mathrm{~cm}$, and $>10 \mathrm{~cm})$. Grosstotal resections (GTRs) were performed in 172 patients ( $81.9 \%$ overall, or $86.5 \%, 79.0 \%$, and $77.5 \%$ in the 3 groups, respectively). Subtotal and partial resections were achieved in 38 patients (18.1\%). Eight patients with medulla oblongata or upper cervical cord tumors received a tracheotomy postoperatively. The follow-up period ranged from 56 to 176 months. One hundred thirty-five patients $(76.7 \%)$ experienced improvement, $(88.2 \%, 83.8 \%$, and $34.4 \%$ in the $<5 \mathrm{~cm}, 5-10 \mathrm{~cm}$, and $>10 \mathrm{~cm}$ groups, respectively). Thirty-three patients (18.8\%) maintained their pretreatment status, and 8 patients $(4.5 \%)$ showed deterioration following tumor resection at 6 months. Tumor recurrence or progression was observed in $6(2.9 \%)$ of the 210 patients. Among the 6 patients, recurrent tumors were located in the conus $(n=3)$, thoracic $(n=1)$, and medullocervical cord $(n=2)$.

Conclusions. Radical resection of spinal ependymomas could be performed in most patients, and the rate of GTR was significantly different in the different-length groups $(<10 \mathrm{~cm} v s>10 \mathrm{~cm}, \mathrm{p}=0.032)$. Patients with longer tumors had worse surgical results compared with those with small tumors $(\mathrm{p}<0.001)$, and more postoperative neuropathic pain and proprioceptive deficits could usually be observed in patients harboring larger tumors. Early diagnosis and timely operation are critical to achieving better neurological outcomes. For tumors with dense adhesions, complete removal should be performed cautiously because of the significant incidence of neurological deterioration. (http://thejns.org/doi/abs/10.3171/2014.3.SPINE13481)

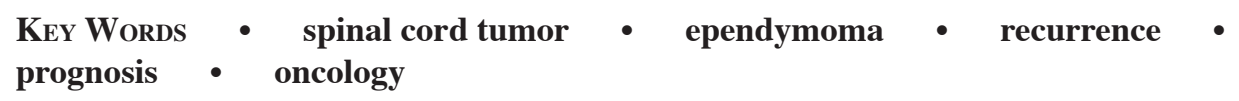

$\mathrm{E}$ PENDYMOMAS are neuroectodermal tumors that can develop in the spinal cord and constitute approximately $30 \%-88 \%$ of primary spinal intramedullary tumors. ${ }^{6}$ Most ependymomas are benign and slow growing, and tend to compress the adjacent cord parenchyma

\footnotetext{
Abbreviations used in this paper: GTR = gross-total resection; $\mathrm{mMEP}=$ muscle motor evoked potential; SSEP = somatosensory evoked potential; STR = subtotal resection.
}

rather than infiltrate it. ${ }^{5,9}$ Such tumors present a relatively clear surgical plane and are curable with gross-total resection (GTR). However, in spite of improved microsurgical techniques, the potential morbidity resulting from surgery can still be formidable even for experienced surgeons. Although GTR is the ultimate goal of surgery,

This article contains some figures that are displayed in color online but in black-and-white in the print edition. 
preservation of spinal cord function should always take priority. Therefore, it is very important to modify surgical plans according to the patient's preoperative status and intraoperative findings.

In this study, we collected retrospective data and documented the length of the solid portion and location of the tumor, neurological function, complications, and recurrence in patients who underwent surgery for spinal ependymomas. The aim of the work was to better define our surgical strategies for performing resection of tumors of different lengths, and more importantly, to analyze functional outcome of these patients with spinal ependymomas in the follow-up period.

\section{Methods}

\section{Study Population}

After obtaining consent from the institutional review board of Beijing Tiantan Hospital, we reviewed the records of patients undergoing surgery for spinal tumors from January 1999 to December 2008. The inclusion criterion was histologically confirmed ependymoma (WHO Grade II) or anaplastic ependymoma (WHO Grade III) with primary spinal localization. Two hundred ten patients with spinal ependymomas were enrolled in this study. The characteristics of the patients were documented, including demographic information, operative details, complications, and tumor recurrence.

\section{Neurological Assessment}

A modified McCormick classification was applied to evaluate the neurological function of the patients. ${ }^{1}$ Grades were assigned on the basis of documented neurological examinations. The functional assessments were conducted at the preoperative stage, postoperative stage, and regular follow-up periods. Improved, stationary, and deteriorated neurological functions were used to assess functional change.

\section{Imaging Study}

Magnetic resonance images of the spine were obtained in patients at admission. The imaging findings included the size of the tumor measured at the solid part with enhancement, presence and length of syringomyelia, and intratumoral hemorrhage. We divided these patients into 3 groups according to the length of the lesions. Follow-up MRI studies were performed in the event of new onset of neurological deficits or for routine postoperative evaluation.

\section{Neurophysiological Monitoring}

Somatosensory evoked potentials (SSEPs) and muscle motor evoked potentials (mMEPs) were used to assist the surgical management of our patients. A $50 \%$ drop in amplitude and/or a greater than $10 \%$ prolongation in latency in SSEP and mMEP disappearance was considered significant in this study.

\section{Surgical Procedures}

The patients were placed in the lateral decubitus po- sition. Using a midline skin incision and laminectomy, the operative field was extended from 1 level above to 1 level below the lesion. Under microscopic assistance a durotomy was performed, and the arachnoid was opened separately. We discriminated the midline of the cord by inspecting the bilateral posterior exiting of spinal nerves and distribution of dorsal vascularity. Then a myelotomy was performed along the midline. The interface between the tumor and the spinal cord was meticulously dissected. We tried to identify the associated syrinx first. The tumor in its entirety was exposed rostrally and caudally, and completely resected in most cases. Ependymomas around the conus medullaris were usually fragile, tenacious, calcified, and rich in blood supply, and the tumors were partially resected. It was also difficult to completely remove lumbosacral tumors with dense adhesion to the cauda equina. In some patients, the surgical strategy was adjusted intraoperatively because of signal changes of evoked potential monitoring. We also used the contact laser as an adjunct in tumor resection to avoid iatrogenic spinal cord injury. After adequate hemostasis, the pia, arachnoid, and dura matter were closed layer by layer. The laminae were put back in situ using titanium plates and screws (laminoplastic laminotomy).

\section{Extent of Tumor Resection}

The extent of surgery was classified as GTR, subtotal resection (STR), or partial resection. Gross-total resection was defined as gross macroscopic removal of the visible tumor or no evidence of a residual tumor on postoperative MRI. Resection was considered subtotal when at least $80 \%$ of the tumor was excised. Partial resection was consistent with a less than $80 \%$ resection.

\section{Tumor Recurrence or Progression}

The clinical and radiological findings were integrated to evaluate tumor recurrence or progression. Reappearance or worsening of neurological symptoms in the patients who underwent GTR implied recurrence, and the diagnosis was established by a follow-up MR image showing a new-growing tumor arising from the vertebral levels spanned by the primary tumor. Progressive disease after subtotal or partial resection was indicated by deterioration of the previously stable neurological signs and symptoms and volumetric progression on radiographic findings.

\section{Statistical Analyses}

The extent of resection, the difference between the preoperative and postoperative modified McCormick grade at discharge, and the follow-up examinations were compared between the different length groups $(<5 \mathrm{~cm}$, $5-10 \mathrm{~cm}$, and $>10 \mathrm{~cm}$ ). We also attempted comparisons between the two age groups (pediatric and adult) and the tumor locations to determine whether they were influential factors in the extent of tumor resection. Differences between groups were evaluated using the Pearson chisquare test. A p value $<0.05$ was considered statistically significant. Analyses were performed using the statistical software package SPSS (version 13, SPSS, Inc.). 


\section{Results}

Among the 210 patients, there were 120 males and 90 females. The age at the time of surgery ranged from 5 to 70 years (Fig. 1). The follow-up period, calculated from the date of surgery to the patient's most recent clinic visit or telephone interview, ranged from 56 to 176 months.

At admission, 47 patients (22.4\%) had McCormick Grade Ib, 95 (45.2\%) had Grade II, 55 (26.2\%) had Grade III, and 13 (6.2\%) had Grade IV neurological function (Table 1). In addition, scoliosis was noted in 21 patients $(10.0 \%)$ and cross-foot abnormality was documented in $11(5.2 \%)$. Twenty-one patients had abnormal pulmonary function tests.

The MR images showed the tumor located in the cervical region in 139 patients $(66.2 \%)$, the thoracic region in $62(29.5 \%)$, and the lumbar region or conus medullaris in $9(4.3 \%)$. The cumulative number of ependymomas at each level is shown in Fig. 2. Tumors were stratified into 3 groups, according to diameter, $<5 \mathrm{~cm}, 5-10 \mathrm{~cm}$, and $>10$ $\mathrm{cm}$, and the number of cases in each group was 89,81 , and 40 , respectively. The presence of syringomyelia was identified in 147 patients $(70 \%)$, and the average length of the syrinx in each group is summarized in Table 1. Twenty-two cases with intratumoral hemorrhage were found by MRI examination.

Total resections were performed in 172 patients $(81.9 \%$ overall, or $86.5 \%, 79.0 \%$, and $77.5 \%$ in the 3 groups, respectively). Subtotal and partial resections were achieved in 38 patients (18.1\%; Table 2). Eight patients with medulla oblongata or upper cervical cord tumors received a tracheotomy postoperatively. During the perioperative period, ileus occurred in 40 patients who were usually older $>60$ years of age) and had thoracic cord tumors. No patient received adjuvant radiotherapy.

After the primary surgery, 120 patients (57.1\%) experienced improvement according to the McCormick classification grade: $59(28.1 \%)$ maintained their pretreatment status and $31(14.8 \%)$ showed deterioration following tumor resection. At the 6-month follow-up, 135 patients $(76.7 \%)$ experienced improvement $(88.2 \%, 83.8 \%$, and $34.4 \%$ in the $<5 \mathrm{~cm}, 5-10 \mathrm{~cm}$, and $>10 \mathrm{~cm}$ groups, respectively), 33 $(18.8 \%)$ maintained their pretreatment status, and 8 (4.5\%)

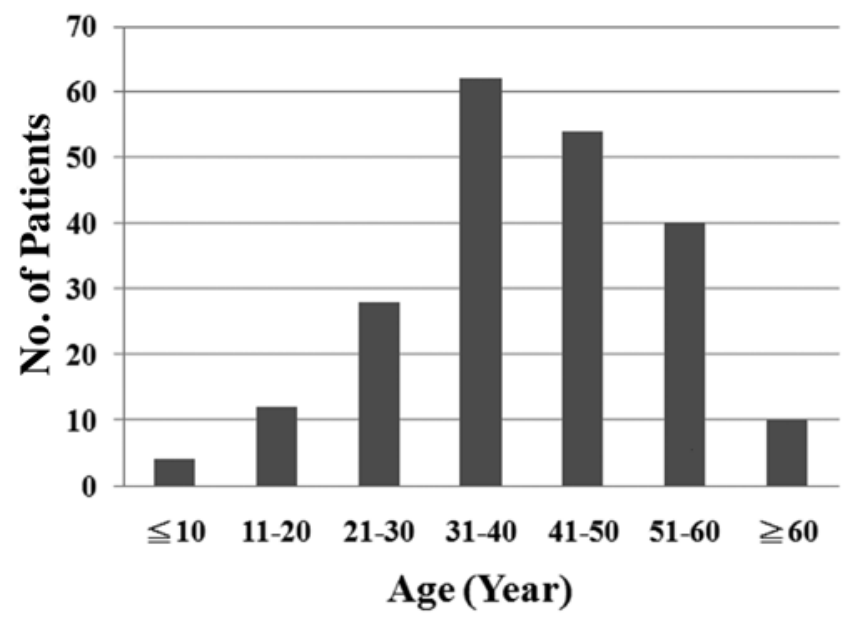

Fıg. 1. Age distribution of 210 cases with spinal ependymomas. showed deterioration following tumor resection (Table 3). The functional status at the final follow-up evaluation is shown in Fig. 3, comparing these results with the functional status at the 6-month evaluation. We combined the $0-5 \mathrm{~cm}$ group and the $5-10 \mathrm{~cm}$ group due to their similar prognoses and compared them with the $>10 \mathrm{~cm}$ group. The prognosis in the $<10 \mathrm{~cm}$ group was better than that in the $>10 \mathrm{~cm}$ group immediately postoperatively and at the 6 -month follow-up ( $p<0.001)$. Table 4 shows the relationship between tumor location and extent of resection; the extent of resection was significantly different at different locations $(p=0.009)$. In the pediatric group we achieved GTR, STR, and partial resection in 6,1 , and 2 patients, respectively, which was comparable to the results in the adult group ( $\mathrm{p}=0.241$ ). Table 3 shows the summary of preoperative McCormick classification and 6-month neurological outcomes. There were 190 cases $(90.5 \%)$ presenting with postoperative sensation deficits (28 cases newly occurred), including numbness, pain, or impaired proprioception, and most patients experienced complete or partial improvement of sensory disturbance within 1 year. Eleven patients (5.2\%) with conus ependymomas developed bowel or bladder dysfunction after the operation. These associated autonomic dysfunctions can usually be recovered in the 1.5year follow-up period. During the follow-up period, several patients developed severe kyphotic deformity without any neurological deterioration (Figs. 4-6). However, most of the patients lacked long-term imaging examinations, so the frequency of postoperative kyphosis was difficult to determine.

Among the 168 patients who underwent intraoperative SSEP and mMEP monitoring, only 155 cases had identifiable SSEPs, and 129 cases had mMEPs. The association between SSEP and mMEP changes and surgical outcome is shown in Tables 5 and 6 . We found that patients with intraoperative SSEPs that declined or disappeared (79/155, $51 \%$ ) tended to have more postoperative sensory dysfunction, mainly numbness, pain, or proprioception dysfunction of the dominant area. These changes may be caused by interference with or damage to posterior column spinal cord tracts when incising the spinal cord or separating the tumor intraoperatively, and sensory dysfunction is difficult to recover postoperatively. A decline or disappearance in mMEP amplitude $(37 / 129,29 \%)$ was difficult to restore even when the operation was aborted, but movement function was gradually restored postoperatively in long-term follow-up up to 2 years. The significant changes of SSEPs and mMEPs correlate well with worse sensory function and lower modified McCormick grade $(\mathrm{p}<0.001)$ at 1 week after the operation and at long-term follow-up.

Tumor recurrence or progression was observed in 6 (2.9\%) of the 210 patients. Two patients with anaplastic ependymoma (WHO Grade III) experienced tumor recurrence, and 1 patient underwent a second operation (Fig. 7). Among the 6 patients, recurrent tumors were located in the conus medullaris $(n=3)$ and in the thoracic $(n=1)$ and medullocervical cord $(n=2)$.

\section{Discussion}

Spinal cord ependymomas are the most common in- 
T. Li et al.

TABLE 1: Summary of characteristics of the 3 ependymoma groups

\begin{tabular}{|c|c|c|c|}
\hline Variable & $<5 \mathrm{~cm}$ & $5-10 \mathrm{~cm}$ & $>10 \mathrm{~cm}$ \\
\hline no. in group & 89 & 81 & 40 \\
\hline mean length of syrinx $(\mathrm{cm})$ & 6.32 & 5.87 & 6.43 \\
\hline mean preop symptom duration (mos) & 18.2 & 44.2 & 30 \\
\hline \multicolumn{4}{|l|}{ preop symptoms (\%) } \\
\hline sensory & $63(70.8)$ & $71(87.7)$ & $36(90.0)$ \\
\hline motor & $57(64.0)$ & 69 (85.2) & $39(97.5)$ \\
\hline sphincter & $21(23.6)$ & $18(22.2)$ & $21(52.5)$ \\
\hline pain & $27(30.3)$ & $51(63.0)$ & $24(60.0)$ \\
\hline \multicolumn{4}{|l|}{ postop new deficit (\%) } \\
\hline sensory & $15(16.9)$ & $9(11.1)$ & $4(10.0)$ \\
\hline motor & $3(3.4)$ & $3(3.7)$ & $1(2.5)$ \\
\hline pain & $3(3.4)$ & $15(18.5)$ & $9(22.5)$ \\
\hline \multicolumn{4}{|l|}{ McCormick preop grade (\%) } \\
\hline $\mathrm{lb}$ & $27(30.3)$ & $18(22.2)$ & $2(5.0)$ \\
\hline II & $41(46.1)$ & $36(44.4)$ & $18(45.0)$ \\
\hline III & $15(16.9)$ & $24(29.6)$ & $16(40.0)$ \\
\hline IV & $6(6.7)$ & $3(3.7)$ & $4(10.0)$ \\
\hline \multicolumn{4}{|l|}{ McCormick immediate postop grade (\%) } \\
\hline I & $9(10.1)$ & $9(11.1)$ & $2(5.0)$ \\
\hline $\mathrm{lb}$ & $27(30.3)$ & $35(43.2)$ & $14(35)$ \\
\hline ॥ & $36(40.4)$ & $22(27.2)$ & $13(32.5)$ \\
\hline III & $12(13.5)$ & $6(7.4)$ & $8(20.0)$ \\
\hline IV & $5(5.6)$ & $9(11.1)$ & $3(7.5)$ \\
\hline \multicolumn{4}{|l|}{ McCormick 6-month postop grade (\%) } \\
\hline I & $37(43.5)$ & $34(44.2)$ & $5(13.2)$ \\
\hline $\mathrm{lb}$ & $33(38.8)$ & $26(33.8)$ & $20(52.6)$ \\
\hline II & $7(8.2)$ & $5(6.5)$ & $8(21.1)$ \\
\hline III & $3(3.5)$ & $3(3.9)$ & $3(7.9)$ \\
\hline IV & $5(5.9)$ & $9(11.7)$ & $2(5.3)$ \\
\hline
\end{tabular}

tramedullary tumors and are usually located centrally. Extramedullary ependymomas are rare ${ }^{8}$ Complete tumor removal can be achieved in such lesions when there are clear surgical boundaries. For prognostic purposes, early diagnosis and surgical treatment are crucial. ${ }^{7}$ If the tumors are bulky or compress the medulla oblongata or upper cervical cord, GTR can be highly risky. ${ }^{3}$

Because most spinal cord ependymomas are slow growing, the clinical progression is obscure. Few patients present with symptoms of acute neurological deterioration resulting from tumor hemorrhage. ${ }^{4}$ In our series, the duration of symptoms was 30.8 months on average, while the $0-5 \mathrm{~cm}$ group had a shorter duration of symptoms than the other two groups (Table 1). The majority of our cases were classified as McCormick Class II or III in the 5-10 $\mathrm{cm}$ and $>10 \mathrm{~cm}$ groups, while the majority of patients were classified as McCormick Class Ib or II in the 0-5 $\mathrm{cm}$ group. There were 5 patients who had tumors more than $20 \mathrm{~cm}$ in length. Figure 4 shows a representative MR image and tumor specimen from 1 of these 5 patients. In addition, 33 cases were initially misdiagnosed as cervical or lumbar spondylosis. Clinicians should strongly suspect intramedullary lesions when patients have abnormal locomotor or sensory functions. Magnetic resonance images with and without contrast enhancement are critical for timely diagnosis. The presence of syringomyelia was identified in 147 patients (70\%) and the average length of the syrinx had no correlation with the length of the tumor.

Ependymomas may be complicated by tumor hemorrhage, which usually leads to rapid neurological worsening. Tumor hemorrhages were identified intraoperatively in 22 cases and can result in dense adhesion between tumor and spinal cord. Hemorrhage may extend beyond the superior and inferior borders of the tumor within the central canal and cause increased pressure on the spinal cord. Therefore, analyzing the details of MR images is important for preoperative planning and risk estimation. A hematoma in different stages shows mixed signals, and marked hypointensity of hemosiderin may be noted (Fig. 8 ). These lesions have uneven and irregular enhancement after contrast administration, and should be distinguished from intramedullary astrocytomas.

Surgical treatment of spinal ependymomas requires careful evaluation of surgical risks in relation to the natural 


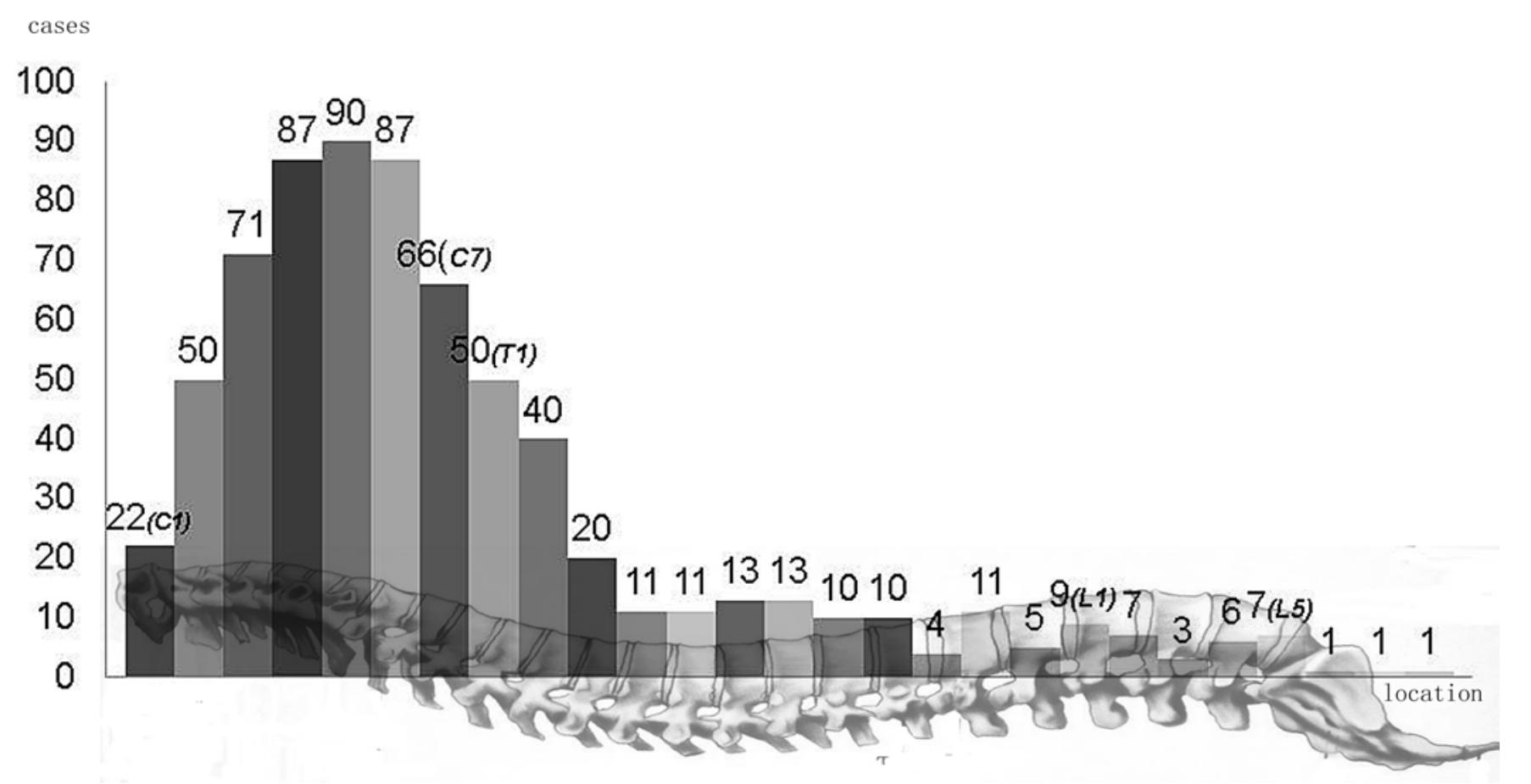

Fig. 2. The cumulative number of ependymomas at each spinal level.

history of intramedullary ependymomas. Complete tumor resection with preservation of as much neural function as possible remains the ultimate goal in clinical practice. In our series, there were 40 intramedullary ependymomas that were more than $10 \mathrm{~cm}$ in length. Because of the high surgical risk and the time required to seek multiple surgical opinions, some patients missed the best surgical treatment window. Sixty-eight patients in our series presented with severe disability and were classified as McCormick Class III or IV. Our results show that the patients in McCormick Class Ib and II have better neurological recovery than those in McCormick Class III and IV. Patients with higher McCormick classes usually had longer tumors and duration of symptoms (Table 1). Thus, proper patient education and early surgical intervention before functional deterioration play an important role in successful therapy.

We found that the percentage of GTR and STR in the 0-5 $\mathrm{cm}$ and 5-10 $\mathrm{cm}$ groups to be similar when using the highly demanding microsurgical technique. In our cases, incomplete tumor resection often resulted from unclear tumor boundaries or profuse intraoperative bleeding. Tumor location can therefore dictate when the resection should be aborted: for tumors located in the medulla oblongata or upper cervical cord, if the tumor is bulky, or closely adherent to the surrounding tissue, a partial resection may be selected if the mMEP disappears intraoperatively or heart rate and blood pressure fall. If the tumors are located in the thoracic spinal cord, the tumor is longer than $10 \mathrm{~cm}$, and the compression of the spinal cord is severe, we usually choose partial resection when the mMEPs shows significant changes, as they are highly correlated with postoperative paralysis. If only the SSEPs shows significant changes, we usually continue the surgery after careful reinspection of the myelotomy site and adjustment of surgical traction. For tumors located in the conus medullaris, we may choose partial resection when defecation dysfunction appears preoperatively in patients or sphincter electromyography demonstrates significant changes. Patients' preoperative neurological status is another important factor when deciding whether complete resection is achievable. If patients have severe respiratory dysfunction, defecation dysfunction, or paralysis preoperatively, we believe that the surgeons should be more conservative. The length of the tumors has a significant

TABLE 2: Summary of preoperative modified McCormick classification and outcome of resection

\begin{tabular}{|c|c|c|c|c|c|c|c|c|c|}
\hline \multirow[b]{2}{*}{$\begin{array}{l}\text { Preop McCormick } \\
\text { Classification }\end{array}$} & \multicolumn{3}{|c|}{$<5 \mathrm{~cm}$} & \multicolumn{3}{|c|}{$5-10 \mathrm{~cm}$} & \multicolumn{3}{|c|}{$>10 \mathrm{~cm}$} \\
\hline & GTR & STR & $\begin{array}{c}\text { Partial } \\
\text { Resection }\end{array}$ & GTR & STR & $\begin{array}{c}\text { Partial } \\
\text { Resection }\end{array}$ & GTR & STR & $\begin{array}{c}\text { Partial } \\
\text { Resection }\end{array}$ \\
\hline $\mathrm{lb}$ & 27 & 0 & 0 & 17 & 1 & 0 & 1 & 0 & 1 \\
\hline$\|$ & 35 & 6 & 0 & 26 & 9 & 1 & 15 & 2 & 1 \\
\hline III & 12 & 3 & 0 & 19 & 3 & 2 & 13 & 1 & 2 \\
\hline IV & 3 & 3 & 0 & 2 & 1 & 0 & 2 & 2 & 0 \\
\hline total & 77 & 12 & 0 & 64 & 14 & 3 & 31 & 5 & 4 \\
\hline
\end{tabular}


TABLE 3: Summary of preoperative modified McCormick classification and 6-month neurological outcomes

\begin{tabular}{|c|c|c|c|c|c|}
\hline \multirow[b]{2}{*}{ Evaluation } & \multicolumn{5}{|c|}{ McCormick Classification } \\
\hline & $\mathrm{lb}$ & $\|$ & III & IV & Total $(\%)$ \\
\hline preop & 37 & 85 & 45 & 9 & 176 \\
\hline \multicolumn{6}{|l|}{ 6-month outcome } \\
\hline \multicolumn{6}{|l|}{$<5 \mathrm{~cm}$} \\
\hline improved & 18 & 35 & 13 & 1 & $67(88.2)$ \\
\hline maintained & 4 & 1 & 2 & 1 & $8(10.5)$ \\
\hline deteriorated & 0 & 0 & 1 & 0 & $1(1.3)$ \\
\hline \multicolumn{6}{|l|}{$5-10 \mathrm{~cm}$} \\
\hline improved & 13 & 31 & 13 & 0 & $57(83.8)$ \\
\hline maintained & 0 & 2 & 3 & 3 & $8(11.8)$ \\
\hline deteriorated & 0 & 0 & 3 & 0 & $3(4.4)$ \\
\hline \multicolumn{6}{|l|}{$>10 \mathrm{~cm}$} \\
\hline improved & 1 & 6 & 4 & 0 & $11(34.4)$ \\
\hline maintained & 1 & 7 & 5 & 4 & $17(53.1)$ \\
\hline deteriorated & 0 & 3 & 1 & 0 & $4(12.5)$ \\
\hline
\end{tabular}

impact on the opportunity to achieve GTR $(<10 \mathrm{~cm}$ vs $>$ $10 \mathrm{~cm}, \mathrm{p}=0.032$ ).

The length of ependymomas is also an important surgical prognostic factor. Larger tumors tend to have poorer clinical outcome than small tumors because of the patients' worse preoperative neurological function $(\mathrm{p}<$ 0.001). Immediately after the surgery, many patients have new postoperative neuropathic pain and proprioceptive deficits. Patients with longer tumors have more postoperative neuropathic pain and more proprioceptive deficits than those with shorter tumors (Table 1). Although many patients have worse neurological function immediately after surgery, most showed significant recovery in 3-6 months. Some of our patients demonstrated functional improvement up to 2 years after surgery.

There continues to be no consensus regarding laminotomy or laminectomy in spinal tumor surgery. In our opinion, laminotomy is essential, especially for tumors with long segmental involvement and in younger patients. The patients in this group are relatively young, and laminotomy can theoretically prevent postoperative instability and lower the incidence of postoperative epidural adhesions. During the follow-up period, most of the 135 cases undergoing laminotomy after tumor resection had no obvious spinal deformity. Rachiokyphosis was only identified in 2 patients even with laminotomy, which implies that postlaminectomy spinal instability may be affected by various factors.

Surgical techniques are also factors affecting neurological outcomes. Dissection along the dorsal median sulcus of the spinal cord is needed to prevent sensory deprivation. ${ }^{11}$ When the spinal cord is distorted by the enlarged tumor, the bilateral posterior roots and the dorsal central vein of the spinal cord are helpful landmarks to identify the
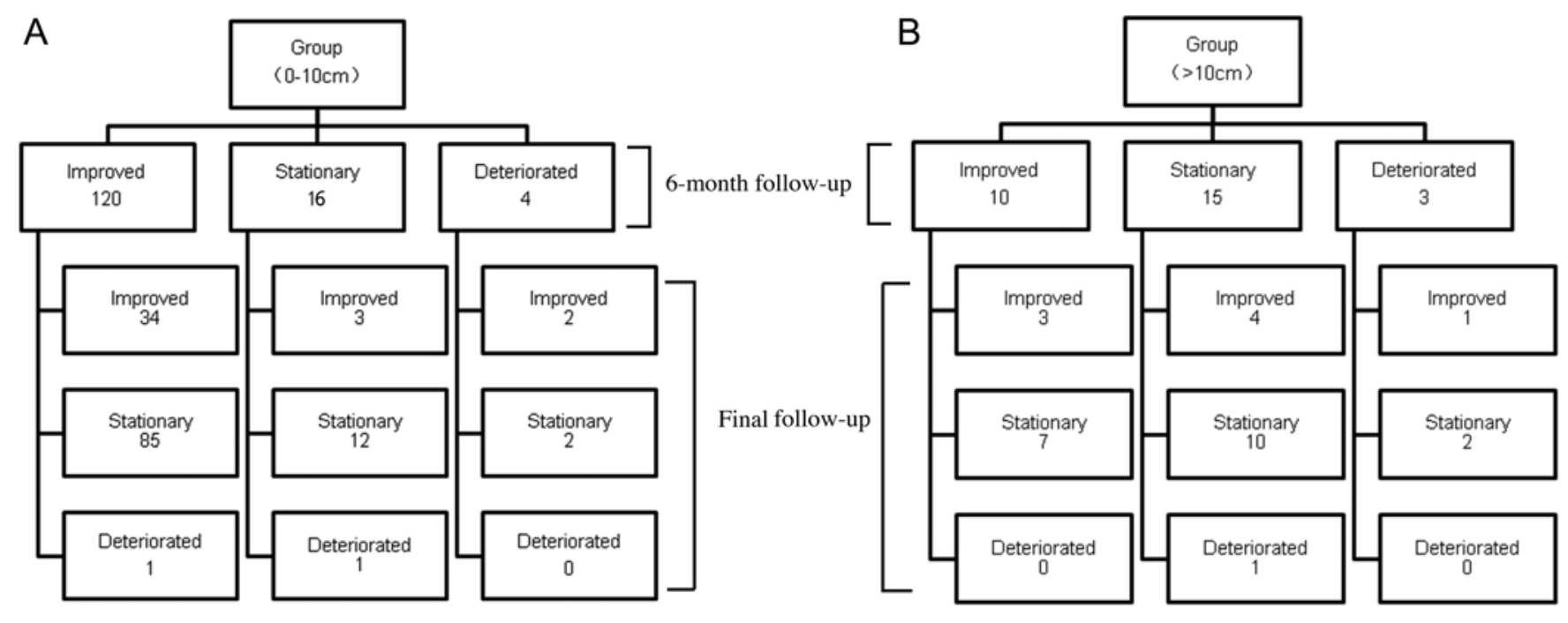

Fig. 3. Illustrations of the changes in neurological function in patients in the $0-10 \mathrm{~cm}$ group (A) and the $>10 \mathrm{~cm}$ group (B) at 6 months and at the last follow-up evaluation. 
Spinal ependymomas of different lengths

TABLE 4: Extent of resection at different locations of the spinal cord

\begin{tabular}{lccc}
\hline & \multicolumn{3}{c}{ Extent of Resection } \\
\cline { 2 - 4 } Spinal Cord Location & Partial & STR & GTR \\
\hline upper cervical & 0 & 12 & 38 \\
lower cervical & 3 & 10 & 76 \\
thoracic & 2 & 6 & 54 \\
conus medullaris & 2 & 3 & 4 \\
\hline
\end{tabular}

midline. In addition, traction of the spinal cord and tumor must be performed carefully. Our methods include fixation of the arachnoid with the dura mater using clips, and suturing the pia with dura mater. In this way, the spinal cord is immobilized, and the tumor can be dissected more easily and safely. If the tumors are poorly defined, we use the contact laser to assist in tumor resection, which reduces the traction of the spinal cord. Ependymomas are hypervascular tumors, and the feeding arteries are usually the branches of the anterior spinal artery as well as the radiculomedullary artery. Long-term tumor expansion results in cord atrophy and an ample blood supply with a consequent increased risk of hemorrhage. Accordingly, bipolar coagu- lation should be performed cautiously, and using absorbable hemostatic gauze or sponges for compression of a small amount of bleeding can avoid injury to the anterior spinal artery. We prefer to remove the tumor in a single block, as piecemeal tumor resection can result in a bloody surgical field and possible tumor spreading. We also temporarily cover tumor beds within the spinal cord with cotton pads to prevent implantation of loose neoplastic cells. When tumors have broken through the ventral surface of the cord, dissections should proceed more carefully as mMEP changes can often occur, caused by either mechanical damage to the thinned and stretched pyramidal tract or ventral vascular compromise. Postoperative adhesion of the spinal cord may be one of the causes of paresthesia. Hence we suture the pia and arachnoid in an interrupted fashion and continuously suture the dura mater separately. We then use laminoplastic laminotomy to prevent epidural adhesion. We routinely use the lateral decubitus position for all spinal tumor surgeries and believe that the lateral position is superior to the prone position, as it allows blood and CSF to drain away from the operative field as gravity pulls the cord tissue down, and facilitates tumor dissection with less cord retraction.

Intraoperative neurophysiological monitoring is able
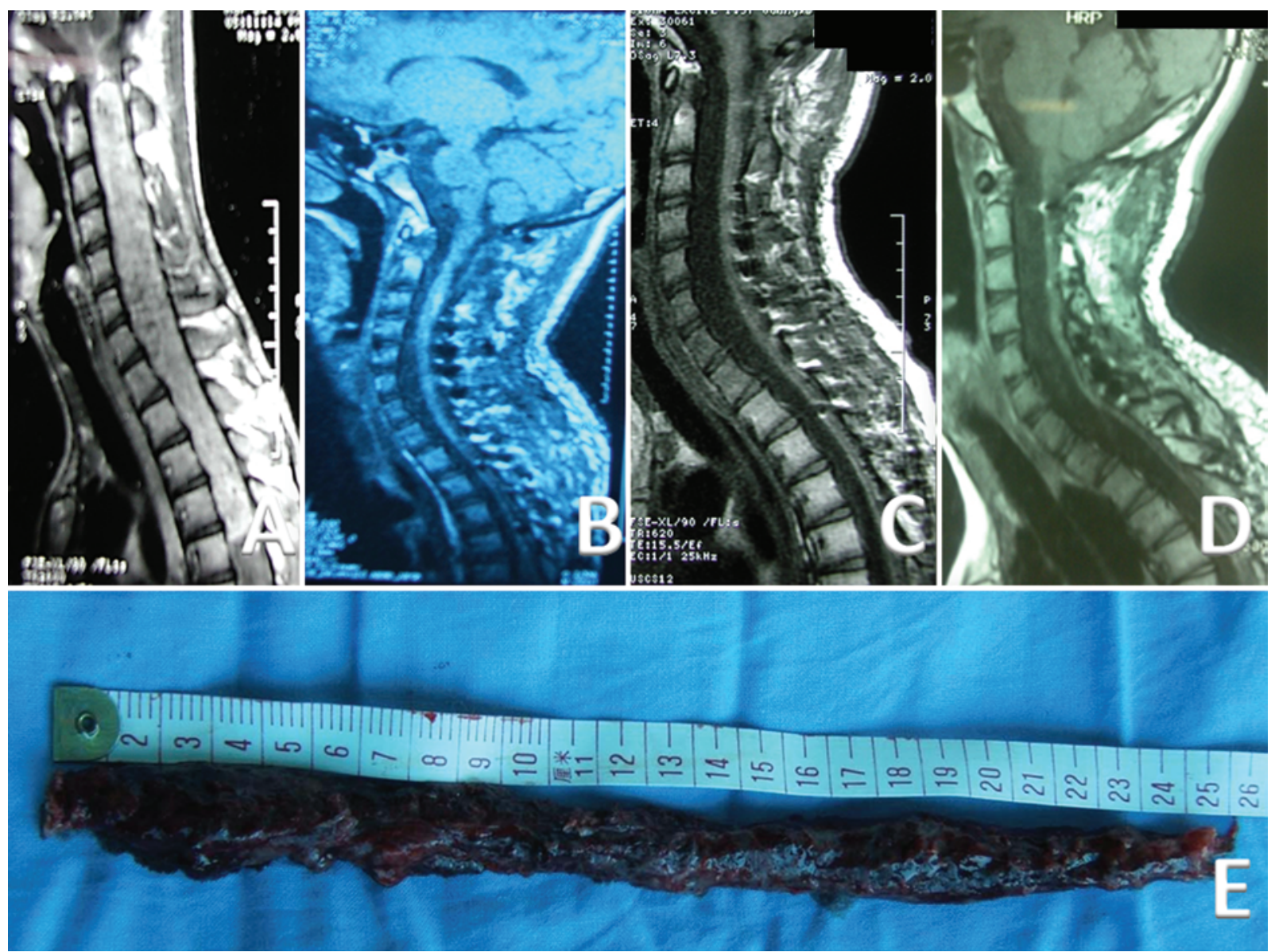

Fig. 4. Images from a patient with an intramedullary ependymoma from the medulla oblongata to T-5. A-D: Sagittal T1weighted MR images after Gd administration, obtained at diagnosis (A), 3 weeks after GTR and laminoplastic laminotomy without change of the lordotic curve (B), 2 years following the operation with early kyphotic change (C), and 5 years after the operation without evidence of recurrence but severe kyphosis (D). E: The tumor specimen showing a length of $26 \mathrm{~cm}$. 

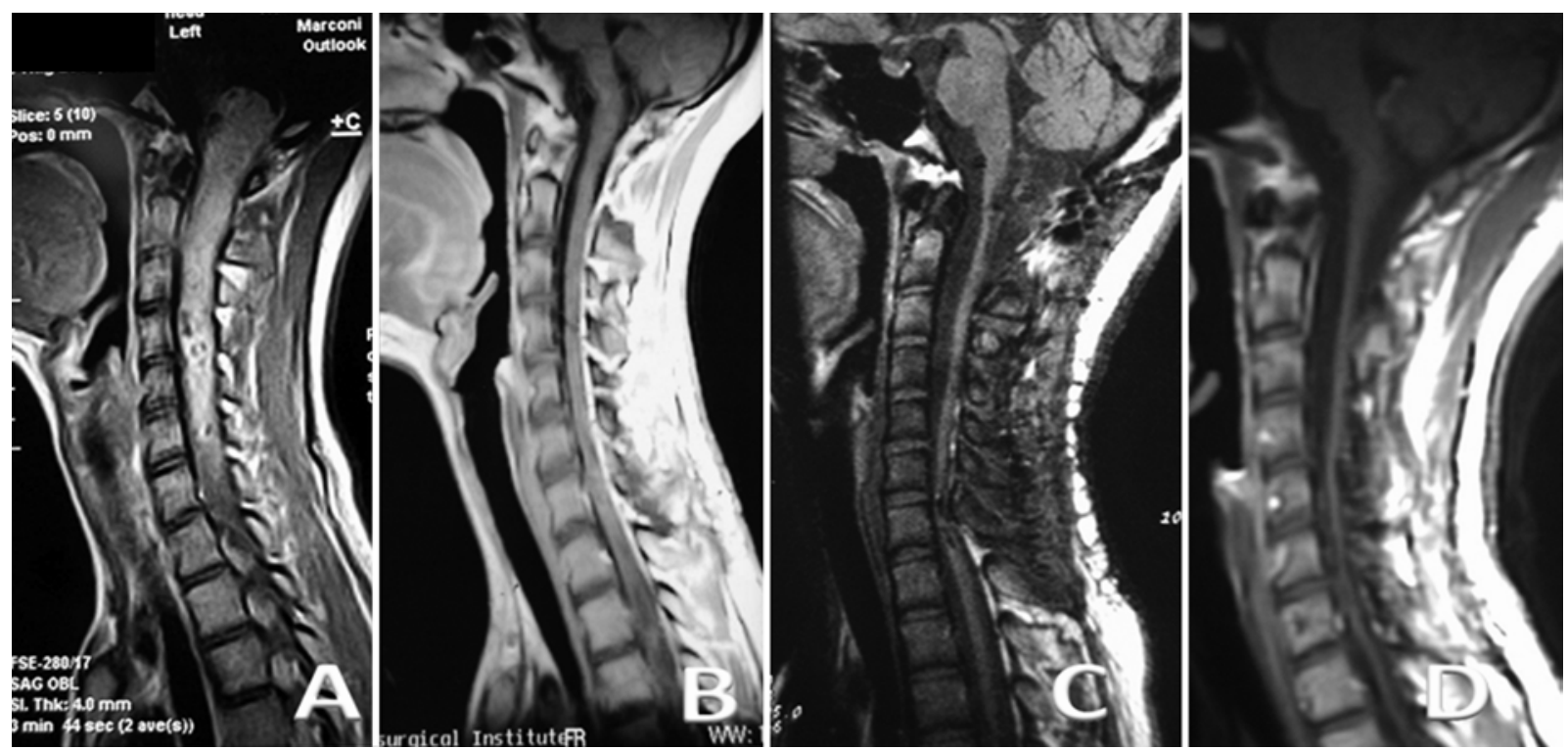

FIG. 5. Images from a patient with an intramedullary ependymoma from the medulla oblongata to T-1. Sagittal T1-weighted MR images after Gd administration at diagnosis (A), 2 weeks after GTR and laminoplastic laminotomy (B), 1 year following the operation (C), and 5 years after the operation without evidence of recurrence or spinal deformity (D).

to provide valuable information for surgeons. When signals of intraoperative monitoring change, surgeons must be cautious and stop the manipulation before irreversible spinal cord injury occurs. Intraoperative mMEPs may be more accurate than other evoked potentials. However, neurophysiological monitoring is not always correlated with postoperative neurological function. As a result, prompt judgment of experienced neurosurgeons is also important for achieving successful tumor resection. Neurological function can improve up to 2 years after surgery.

The patients with medulla oblongata or upper cord ependymomas may have respiratory sequelae resulting from ineffective central respiratory drive, ventilatory
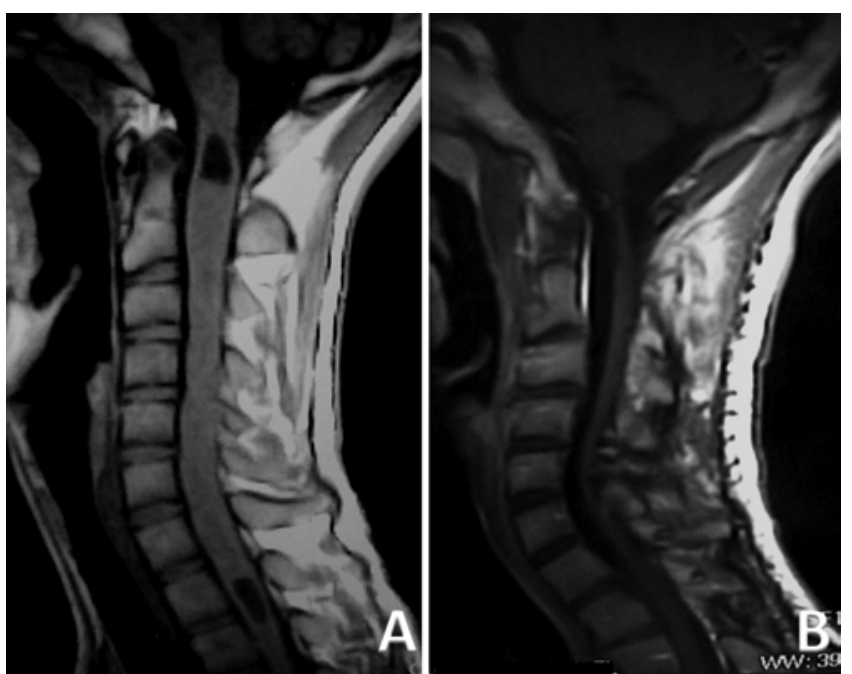

FIG. 6. Sagittal T1-weighted MR images of a C1-T1 intramedullary ependymoma in a 36-year-old male patient (with an allergy to contrast media). Images show an isointense tumor with the presence of a syrinx at the cranial and caudal ends at diagnosis $(A)$ and 5 years after GTR and laminoplastic laminotomy without evidence of recurrence (B). muscle weakness, or retained secretions. Preoperative respiratory function must be carefully evaluated. Postoperatively, hyperbaric oxygenation is needed as early as possible. If respiratory decompensation occurs, patients require intubation with the assistance of mechanical ventilators. Eventually some of these patients require a tracheostomy to facilitate airway management and the removal of excess pulmonary secretions. hygiene. ${ }^{10}$

Unfortunately, even after technically successful surgical excision, significant clinical improvements rarely occur in patients with severe or long-standing preoperative neurological deficits. ${ }^{2}$ Preservation rather than restoration of neurological function is the reasonable aim of surgery. When adhesion between tumor and spinal cord is strong, GTR of the tumor is not feasible (Fig. 9). We recommend a regular follow-up schedule and consider a second operation for tumor recurrence or progression. The authors found that radiation therapy cannot effectively control tumor growth, but can cause serious neurological damage and greater challenges for a second surgery. Therefore, we do not routinely recommend radiation therapy for patients with incomplete resection.

\section{Conclusions}

Radical resection of spinal ependymomas can be per-

TABLE 5: Relationship between SSEP monitoring results and sensory function

\begin{tabular}{lccc}
\hline \multirow{2}{*}{\multicolumn{1}{c}{ SSEP }} & \multicolumn{3}{c}{ Sensory Function } \\
\cline { 2 - 4 } changed (1 wk) & Improvement & Maintained & Deteriorated \\
changed (1 yr) & 6 & 51 & 28 \\
unchanged (1 wk) & 9 & 46 & 24 \\
unchanged (1 yr) & 19 & 62 & 5 \\
\hline
\end{tabular}


Spinal ependymomas of different lengths

TABLE 6: Relationship between mMEP monitoring and neurological function

\begin{tabular}{ccccc}
\hline & \multicolumn{4}{c}{ Neurological Function } \\
\cline { 2 - 5 } mMEP & Improvement & Maintained & Moderately Deteriorated & Significantly Deteriorated \\
\hline absence $(1 \mathrm{wk})$ & 1 & 8 & 24 & 4 \\
absence $(2 \mathrm{yrs})$ & 5 & 12 & 17 & 1 \\
presence $(1 \mathrm{wk})$ & 29 & 61 & 2 & 0 \\
presence $(2 \mathrm{yrs})$ & 46 & 41 & 1 & 0 \\
\hline
\end{tabular}

formed in most patients, and the rate of GTR is significantly different in the different-length groups $(<10 \mathrm{~cm}$ $\mathrm{vs}>10 \mathrm{~cm} ; \mathrm{p}=0.032)$. Patients with longer tumors have worse surgical outcomes than those with smaller tumors $(\mathrm{p}<0.001)$, and greater postoperative neuropathic pain and proprioceptive deficits are usually noted in patients harboring larger tumors. The extent of resection was also significantly different at different locations $(\mathrm{p}=0.009)$.
The extent of resection completed in pediatric patients was similar to that in the adult patients $(\mathrm{p}=0.241)$. The significant changes in SSEPs and mMEPs correlate well with postoperative sensory dysfunction and lower modified McCormick grade at 1 week after surgery and at long-term follow-up. Early diagnosis and a timely operation are critical for better neurological outcomes in these patients. For tumors with dense adhesion, complete re-
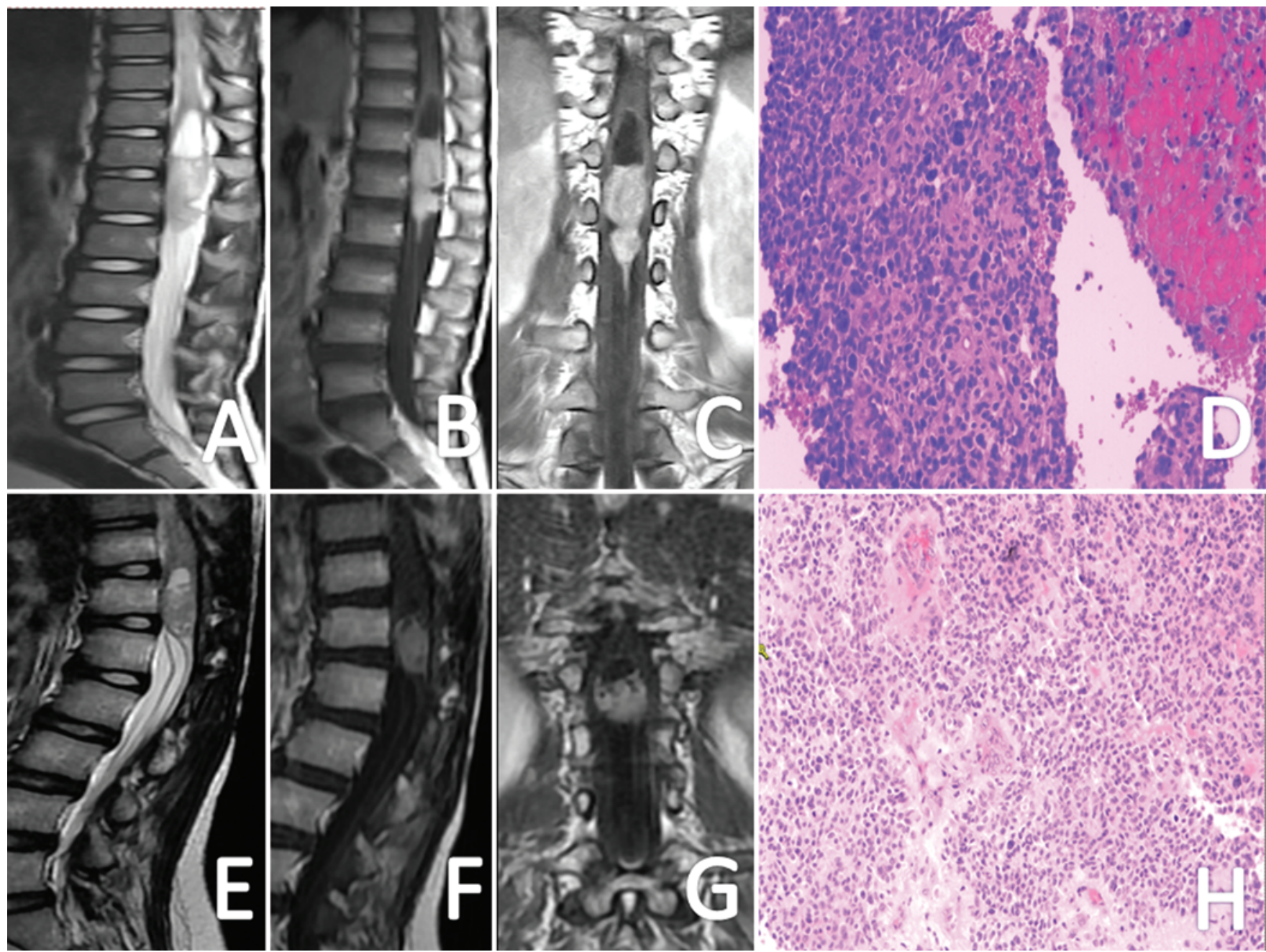

FIG. 7. Images showing characteristics of a T12-L2 ependymoma in an 8-year-old female patient. A sagittal T2-weighted MR image (A) shows an isointense tumor with the presence of a syrinx, and sagittal (B) and coronal (C) T1-weighted MR images obtained after $\mathrm{Gd}$ administration show strong homogeneous enhancement. A photomicrograph obtained after the first operation (D) shows characteristics of an anaplastic ependymoma. $\mathrm{H} \& \mathrm{E}$, original magnification $\times 100$. A sagittal T2-weighted MR image (E) and sagittal (F) and coronal (G) T1-weighted MR images obtained after Gd administration show tumor recurrence 3 years after the operation. A photomicrograph obtained after the second operation $(\mathrm{H})$ also shows characteristics of an anaplastic ependymoma. $H \& E$, original magnification $\times 100$. 
T. Li et al.

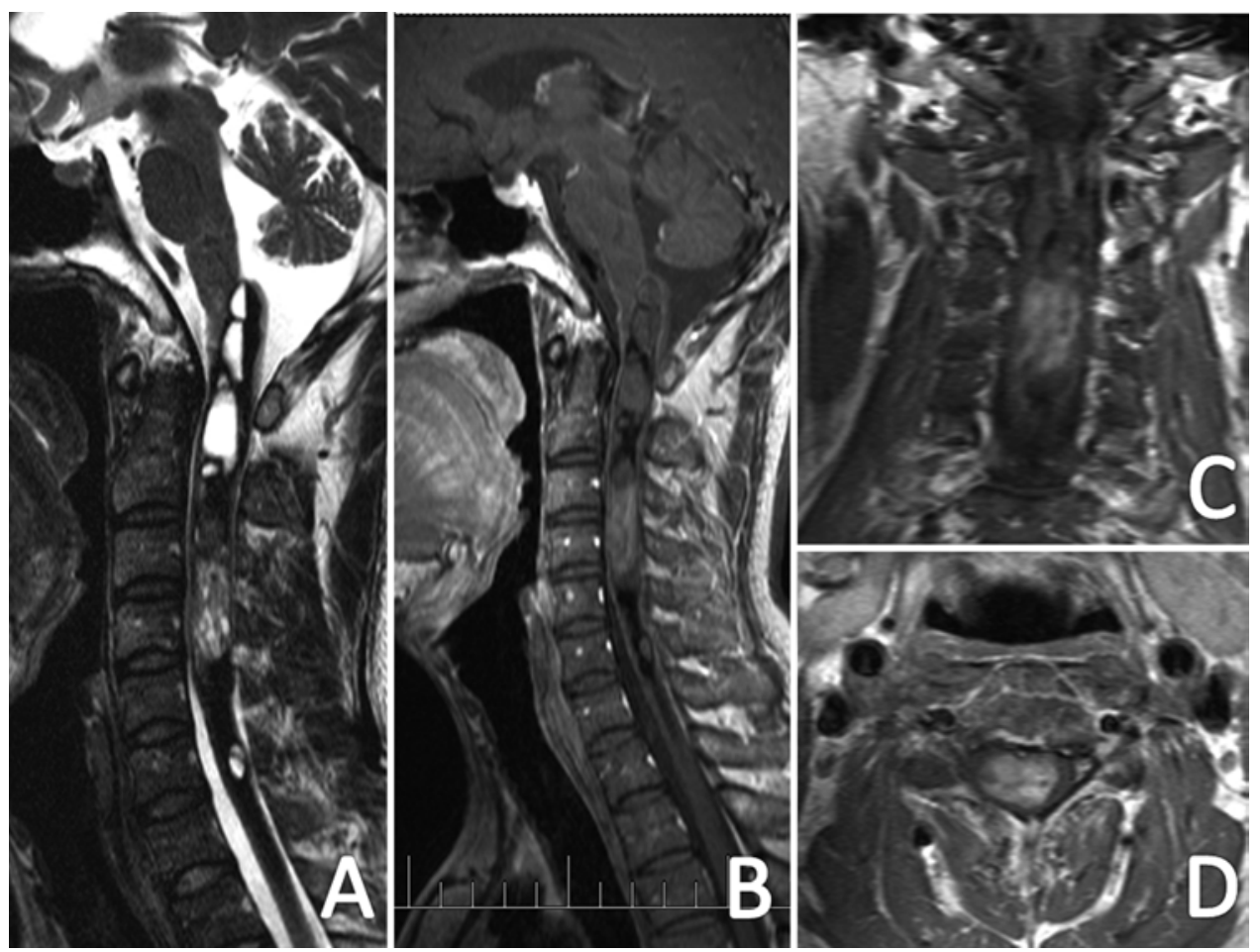

FIG. 8. Images obtained at diagnosis of an intramedullary ependymoma from the medulla oblongata to C-6. A sagittal T1weighted MR image (A) and sagittal (B), coronal (C), and axial (D) T1-weighted MR images after Gd administration show multiple irregular hyperintense and hypointense masses inside the tumor mass.
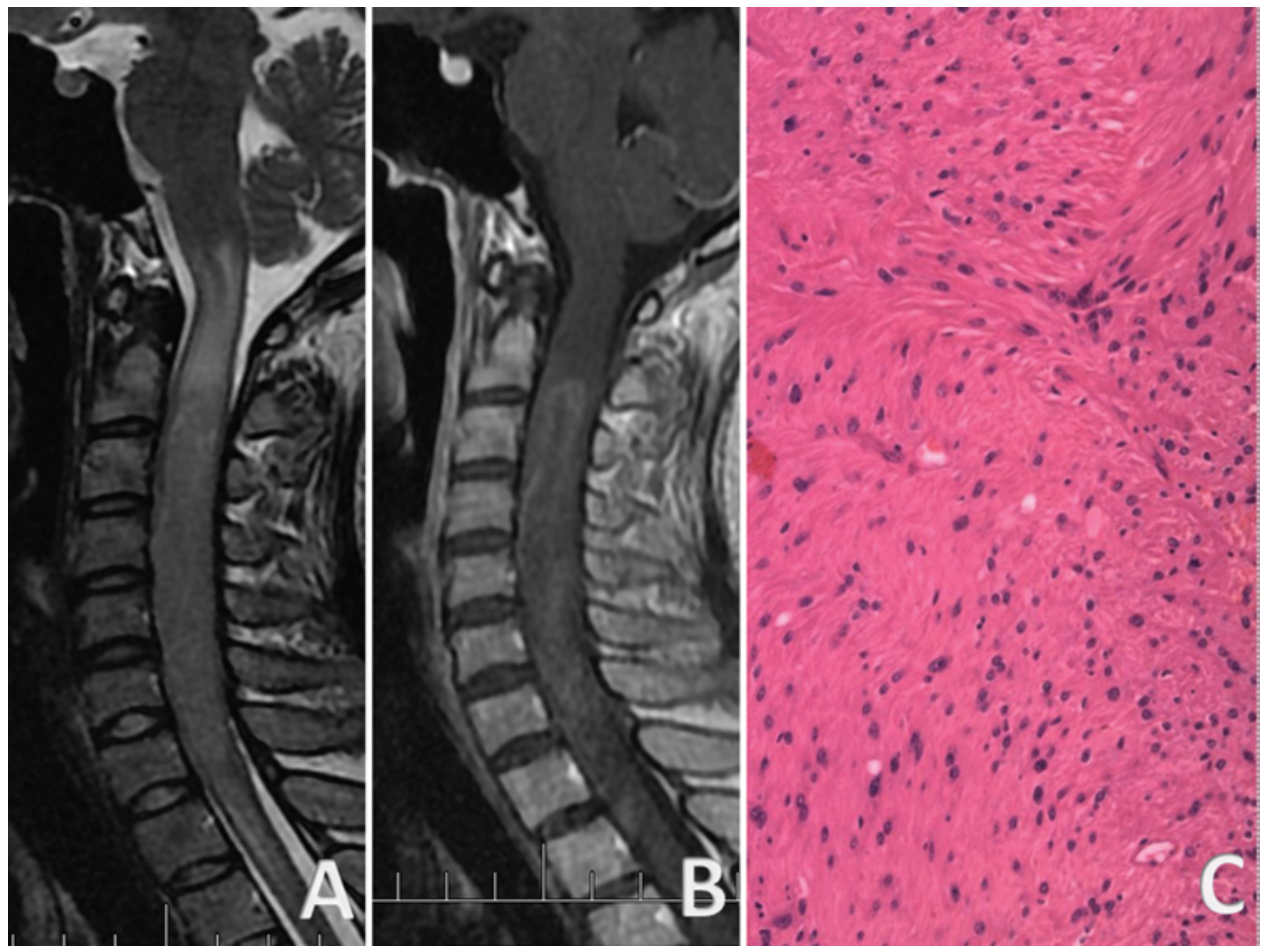

FIG. 9. Images from a patient with an intramedullary ependymoma from $\mathrm{C}-1$ to $\mathrm{T}-2$ with a preoperative diagnosis of an astrocytoma. Sagittal T2-weighted MR images demonstrate a slight hyperintense tumor mass (A) and slight heterogeneous enhancement with an unclear boundary after contrast injection (B). A postoperative photomicrograph (C) confirmed the mass to be a tanycytic ependymoma. $\mathrm{H} \& \mathrm{E}$, original magnification $\times 400$. 


\section{Spinal ependymomas of different lengths}

moval should be performed cautiously because of the significant incidence of neurological deterioration.

\section{Disclosure}

The authors report no conflict of interest concerning the materials or methods used in this study or the findings specified in this paper.

Author contributions to the study and manuscript preparation include the following. Conception and design: G Wang, J Wang, Huang. Acquisition of data: G Wang, Chu, Xu, Yang. Analysis and interpretation of data: Chu, Yang, J Wang, Huang. Drafting the article: G Wang, Li, Huang. Critically revising the article: all authors. Reviewed submitted version of manuscript: all authors. Approved the final version of the manuscript on behalf of all authors: G Wang. Statistical analysis: Li. Administrative/technical/material support: $\mathrm{G}$ Wang, Chu. Study supervision: G Wang.

\section{References}

1. Aghakhani N, David P, Parker F, Lacroix C, Benoudiba F, Tadie M: Intramedullary spinal ependymomas: analysis of a consecutive series of 82 adult cases with particular attention to patients with no preoperative neurological deficit. Neurosurgery 62:1279-1286, 2008

2. Chang UK, Choe WJ, Chung SK, Chung CK, Kim HJ: Surgical outcome and prognostic factors of spinal intramedullary ependymomas in adults. J Neurooncol 57:133-139, 2002

3. de Divitiis E, Spaziante R, Stella L: Giant intramedullary ependymoma. A case report. Neurochirurgia (Stuttg) 21:6972,1978

4. Heuer GG, Stiefel MF, Bailey RL, Schuster JM: Acute paraparesis from hemorrhagic spinal ependymoma: diagnostic dilemma and surgical management. Report of two cases and review of the literature. J Neurosurg Spine 7:652-655, 2007

5. Hoshimaru M, Koyama T, Hashimoto N, Kikuchi H: Results of microsurgical treatment for intramedullary spinal cord ependymomas: analysis of 36 cases. Neurosurgery 44:264-269, 1999

6. Kleihues P, Sobin LH: World Health Organization classification of tumors. Cancer 88:2887, 2000

7. Quigley DG, Farooqi N, Pigott TJ, Findlay GF, Pillay R, Buxton $\mathrm{N}$, et al: Outcome predictors in the management of spinal cord ependymoma. Eur Spine J 16:399-404, 2007

8. Robles SG, Saldana C, Boto GR, Martinez A, Zamarron AP, Jorquera M, et al: Intradural extramedullary spinal ependymoma: a benign pathology? Spine (Phila Pa 1976) 30:E251E254, 2005

9. Schwartz TH, McCormick PC: Intramedullary ependymomas: clinical presentation, surgical treatment strategies and prognosis. J Neurooncol 47:211-218, 2000

10. Zhongcheng W, Guihuai W, Junting Z: [Aggressive surgery for intramedullary upper cord tumors.] Chinese J Neurosurg 14:30-33, 1998 (Chinese)

11. Zhongcheng W, Junting Z, Shaohua Y: [Surgical treatment of intramedullary spinal cord tumors.] Chinese J Neurosurg 13:128-134, 1997 (Chinese)

Manuscript submitted May 25, 2013.

Accepted March 10, 2014.

Please include this information when citing this paper: published online May 16, 2014; DOI: 10.3171/2014.3.SPINE13481.

Address correspondence to: Gui-Huai Wang, M.D., Ph.D., Department of Neurosurgery, Beijing Tiantan Hospital, Capital Medical University, Tiantan Xili 6, Chongwen District, Beijing 100050, China.email: wghwynsby@yahoo.com. 